PROCEEDINGS OF THE

AMERICAN MATHEMATICAL SOCIETY

Volume 134, Number 12, December 2006, Pages 3487-3494

S 0002-9939(06)08001-4

Article electronically published on June 27, 2006

\title{
BASIS PROPERTIES OF EIGENFUNCTIONS OF THE $p$-LAPLACIAN
}

\author{
PAUL BINDING, LYONELL BOULTON, JAN ČEPIČKA, PAVEL DRÁBEK, \\ AND PETR GIRG \\ (Communicated by Carmen C. Chicone)
}

\begin{abstract}
For $p \geqslant \frac{12}{11}$, the eigenfunctions of the non-linear eigenvalue problem for the $p$-Laplacian on the interval $(0,1)$ are shown to form a Riesz basis of $L_{2}(0,1)$ and a Schauder basis of $L_{q}(0,1)$ whenever $1<q<\infty$.
\end{abstract}

\section{INTRODUCTION}

Generalized sine functions $S_{p}, 1<p<\infty$, were studied by Elbert [6] as Dirichlet eigenfunctions for the one-dimensional $p$-Laplacian equation

$$
-\left(y^{\prime}(t)\left|y^{\prime}(t)\right|^{p-2}\right)^{\prime}=(p-1) \lambda y(t)|y(t)|^{p-2}
$$

on $0 \leqslant t \leqslant \pi_{p}$. Here

$$
\pi_{p}:=\frac{2 \pi}{p \sin (\pi / p)}
$$

is the first zero of $S_{p}$, which is the eigenfunction (normalized by $S_{p}^{\prime}(0)=1$ ) corresponding to the minimal Dirichlet eigenvalue $\lambda=1$ of (1). Elbert also deduced the relation

$$
\left|S_{p}\right|^{p}+\left|S_{p}^{\prime}\right|^{p}=1
$$

and considered the higher-order eigenfunctions, which are given by $S_{p}(n t)$ with corresponding eigenvalues $\lambda=n^{p}, n=2,3, \ldots$. When $p=2$, (11) corresponds to Fourier's equation, $S_{2}(t)=\sin (t), \pi_{2}=\pi$ and (3) is the Pythagorean relation.

In [15, Ôtani examined analogous functions $\sin _{p}$ with the factor $(p-1)$ on the right side of (11) replaced by 1 . Lindqvist 12, 13, has studied the $\sin _{p}$ functions in some detail, noting that hyperbolic versions were introduced as early as 1879 . We remark that the $S_{p}$ and $\sin _{p}$ functions have become standard tools in the analysis of more complicated equations with various applications; cf. e.g. [1, 2, 3, 4, [5], 6], 7], 8, 14] and [16].

Received by the editors May 5, 2004 and, in revised form, October 19, 2004.

2000 Mathematics Subject Classification. Primary 34L30; Secondary 34L10, 42A65.

Key words and phrases. $p$-Laplacian eigenvalues, eigenfunction completeness.

The research of the first author was supported by I. W. Killam Foundation and NSERC of Canada.

The second author was supported by a PIMS Postdoctoral Fellowship at the University of Calgary.

The research of the third, fourth, and fifth authors was supported by GAČR, no. 201/03/0671. 
Despite this activity, it seems that analogues for $1<p \neq 2$ of the standard completeness and expansion theorems for sine functions have not been discussed previously. While the $S_{p}$ and $\sin _{p}$ functions can easily be transformed into each other, it turns out that (3) must be modified when $S_{p}$ is replaced by $\sin _{p}$. Since relation (3) simplifies our arguments below, we shall work with $S_{p}$ normalized to the interval $[0,1]$. To this end, we define

$$
f_{n}(t):=S_{p}\left(n \pi_{p} t\right), \quad n \in \mathbb{N},
$$

which comprise eigenfunctions of the nonlinear eigenvalue problem (1) on $(0,1)$ with $y(0)=y(1)=0$, corresponding to eigenvalues $\lambda_{n}=\left(n \pi_{p}\right)^{p}, n=1,2, \ldots$ They admit characterizations, e.g., via a modification of Prüfer's transformation [6] and via Ljusternik-Schnirelmann variational formulae [2].

The functions $f_{n}$ depend on $p$, and in the case $p=2$ they become

$$
e_{n}(t):=\sin (n \pi t), \quad n \in \mathbb{N},
$$

which are proportional to a standard orthonormal basis of the Hilbert space $L_{2}(0,1)$ and form a Schauder basis of the Banach space $L_{q}(0,1)$ for all $q>1$ (see the proof of Theorem 6 below).

Recall that $\left\{g_{n}\right\}$ is a Schauder basis [17, p. 152] of $L_{q}(0,1), 1<q<\infty$, if for any $g \in L_{q}(0,1)$, there exist unique coefficients $c_{n}$, depending continuously on $g$, so that

$$
\left\|\sum_{n=1}^{N} c_{n} g_{n}-g\right\|_{q} \rightarrow 0
$$

as $N \rightarrow \infty$. For $q=2$, such a basis is a Riesz basis [10, p. 74] if the $\ell_{2}$ norm of the above coefficients $c_{n}$ generates a norm equivalent to that in $L_{2}(0,1)$.

The aim of this note is to establish the following.

Theorem 1. For $\frac{12}{11} \leqslant p<\infty$, the family $\left\{f_{n}\right\}_{n=1}^{\infty}$ forms a Riesz basis of $L_{2}(0,1)$ and a Schauder basis of $L_{q}(0,1)$ whenever $1<q<\infty$.

Our main device is a linear mapping $T$ of the space $L_{q}(0,1)$, satisfying $T e_{n}=$ $f_{n}, n \in \mathbb{N}$, and decomposing into a linear combination of certain isometries. In Section 3 we show that $T$ is a bounded operator for $1<p<\infty$. In Section 4 we prove boundedness of the inverse for the range $\frac{12}{11} \leqslant p<\infty$.

We should point out that the bound $\frac{12}{11}$ for $p$ is certainly not optimal, although it is convenient for explicit calculations. We shall make some remarks at the end about this bound, but we note here that our methods will not reduce it to 1 .

\section{Preliminaries}

In this section we describe some elementary properties of $\pi_{p}$ and $S_{p}$, which will be employed in the sequel. We start with

Lemma 2. The number $\pi_{p}$ in (2) is strictly decreasing in $p>1$.

Indeed, a simple computation gives $\frac{d \pi_{p}}{d p}<0$ whenever $p>1$. Notice that $\pi_{p} \nearrow \infty$ as $p \searrow 1$ and $\pi_{p} \searrow 2$ as $p \nearrow \infty$. We shall also need the specific value

$$
\pi_{12 / 11}=\frac{11 \pi \sqrt{2}}{3(\sqrt{3}-1)} .
$$



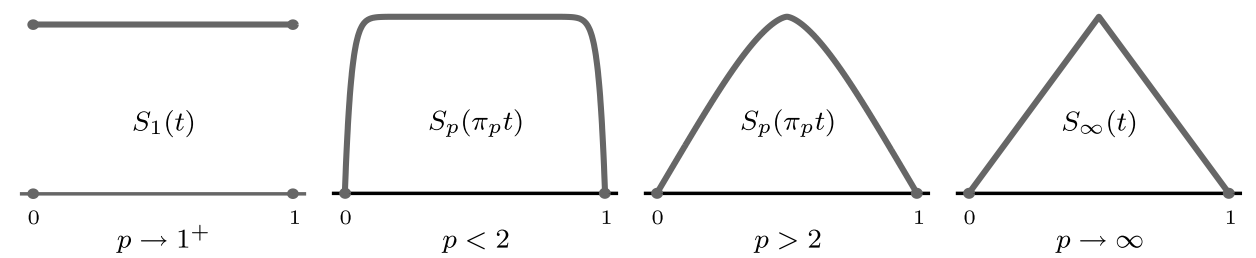

FIGURE 1.

Let $x$ be an arbitrary point in the interval $\left(0, \pi_{p} / 2\right)$. By virtue of (3), $0 \leqslant$ $S_{p}(x) \leqslant 1$ and

$$
S_{p}^{\prime}(x)=\left(1-S_{p}(x)^{p}\right)^{1 / p}>0 .
$$

Furthermore, by construction, $S_{p}(0)=0$ and $S_{p}\left(\pi_{p} / 2\right)=1$.

Lemma 3. Let $p>1$. Then $S_{p}$ is strictly concave on $\left(0, \pi_{p} / 2\right)$, and, when $p \leq 2$,

$$
\left|S_{p}^{\prime \prime}(x)\right| \leqslant 1 .
$$

Proof. By differentiating (5), one readily sees that

$$
S_{p}^{\prime \prime}(x)=-h\left(S_{p}(x)\right)
$$

where $h(y):=y^{p-1}\left(1-y^{p}\right)^{\frac{2-p}{p}}>0$ for $y \in(0,1)$. When $p \leq 2$ it is clear that $|h(y)| \leq 1$.

Remark 4. The above results apply to $f_{1}: t \mapsto S_{p}\left(\pi_{p} t\right)$ on $\left[0, \frac{1}{2}\right]$. We extend $f_{1}$ symmetrically across $\frac{1}{2}$ by $f_{1}(1-t)=f_{1}(t)$. When $p \leq 2$, it is easily seen that Lemma 3 extends to this situation, and so $f_{1}^{\prime \prime}$ is continuous on $(0,1)$. When $p>2$, $f_{1}^{\prime \prime}\left(\frac{1}{2}\right)$ does not exist, but

$$
\int_{0}^{1 / 2}\left|f_{1}^{\prime \prime}(t)\right| \mathrm{d} t=-\pi_{p}^{2} \int_{0}^{1 / 2} S_{p}^{\prime \prime}\left(\pi_{p} t\right) \mathrm{d} t=-\pi_{p}\left[S^{\prime}\left(\pi_{p} t\right)\right]_{t=0}^{1 / 2}=\pi_{p},
$$

and so $f_{1}^{\prime \prime} \in L_{1}(0,1)$.

For any function $f$ on $[0,1]$, we define its successive antiperiodic extension $f^{*}$ over $\mathbb{R}_{+}$by $f^{*}=f$ on $[0,1]$, and

$$
f^{*}(t)=-f^{*}(2 k-t) \text { if } k \leq t \leq k+1, \quad k \in \mathbb{N} .
$$

Then we extend $f_{1}$ over $\mathbb{R}_{+}$via $f_{1}^{*}$, in agreement with $[\underline{6}$.

Finally we need a monotonicity property of this function.

Lemma 5. For each $t \in(0,1), f_{1}(t)=S_{p}\left(\pi_{p} t\right)$ is strictly decreasing in $p>1$.

Proof. From (3), $f_{1}^{-1}(y)=\left(\pi_{p}\right)^{-1} \int_{0}^{y}\left(1-\tau^{p}\right)^{-1 / p} \mathrm{~d} \tau$, for $0 \leqslant y \leqslant 1$. Then the proof follows from the fact that $\left(\pi_{p}\right)^{-1}$ and $\left(1-\tau^{p}\right)^{-1 / p}$ are both positive and strictly increasing in $p>1$ for fixed $\tau \in(0,1)$.

For the reader's convenience, we depict typical profiles of $S_{p}\left(\pi_{p} t\right)$, together with the limiting cases $p=1$ and $p=\infty$, in Figure 1 . 


\section{ThE OPERATOR $T$}

We start by justifying the route we shall take in proving Theorem 1 .

Theorem 6. If there is a linear homeomorphism $T$ of $L_{q}(0,1)$ satisfying $T e_{n}=$ $f_{n}, n \in \mathbb{N}$, for $\frac{12}{11} \leqslant p<\infty$ and $1<q<\infty$, then Theorem 1 holds.

Proof. By [17, Example 11.1, pp. 342-345], any function in $L_{q}(-1,1)$ has a unique sine-cosine series representation. For any $f \in L_{q}(0,1)$, we can thus represent its odd extension to $L_{q}(-1,1)$ uniquely in a sine series, so the $e_{n}$ form a basis of $L_{q}(0,1)$. According to [10, p. 75] the same is true for the $f_{n}$, and so by [17, Theorem 3.1, p. $20]$ they form a Schauder basis of $L_{q}(0,1)$. The argument for a Riesz basis when $q=2$ is similar and follows, e.g., [9, Section VI.2].

In the remainder of this section we define $T$ as a linear combination of certain isometries of $L_{q}(0,1)$. Then we show that $T$ is a bounded operator satisfying $T e_{n}=f_{n}, n \in \mathbb{N}$, for all $p, q>1$.

As in the above proof, the $f_{n}$ (which belong to $L_{q}(0,1)$ ) have Fourier sine series expansions

$$
f_{n}(t)=\sum_{k=1}^{\infty} \widehat{f_{n}}(k) \sin (k \pi t), \text { where } \widehat{f_{n}}(k):=2 \int_{0}^{1} f_{n}(t) \sin (k \pi t) \mathrm{d} t .
$$

An argument involving symmetry with respect to the midpoint $x=\frac{1}{2}$ easily shows that $\widehat{f}_{1}(k)=0$ whenever $k$ is even. Furthermore

$$
\begin{aligned}
\widehat{f_{n}}(k) & =2 \int_{0}^{1} f_{1}(n t) \sin (k \pi t) \mathrm{d} t \\
& =2 \sum_{m \text { odd }} \widehat{f}_{1}(k) \int_{0}^{1} \sin (m \pi n t) \sin (k \pi t) \mathrm{d} t \\
& = \begin{cases}\widehat{f}_{1}(m) & \text { if } m n=k \text { for some } m \text { odd } \\
0 & \text { otherwise. }\end{cases}
\end{aligned}
$$

In what follows we will often denote $\widehat{f}_{1}(j)$ by $\tau_{j}$. We first find a bound on $\left|\tau_{j}\right|$ which will be crucial in the definition of $T$ below. Integration by parts ensures that

$$
\begin{aligned}
& \tau_{j}=2 \int_{0}^{1} f_{1}(t) \sin (j \pi t) \mathrm{d} t=4 \int_{0}^{1 / 2} f_{1}(t) \sin (j \pi t) \mathrm{d} t \\
= & \frac{4}{j \pi} \int_{0}^{1 / 2} f_{1}^{\prime}(t) \cos (j \pi t) \mathrm{d} t=-\frac{4}{j^{2} \pi^{2}} \int_{0}^{1 / 2} f_{1}^{\prime \prime}(t) \sin (j \pi t) \mathrm{d} t,
\end{aligned}
$$

where the integrals exist because $f_{1}^{\prime \prime} \in L_{1}(0,1)$ by Remark 4 . In fact (8) shows that

so

$$
\int_{0}^{1 / 2}\left|f_{1}^{\prime \prime}(t) \sin (j \pi t)\right| \mathrm{d} t<\int_{0}^{1 / 2}\left|f_{1}^{\prime \prime}(t)\right| \mathrm{d} t=\pi_{p}
$$

$$
\left|\tau_{j}\right|<\frac{4 \pi_{p}}{j^{2} \pi^{2}}, \quad \text { for all } p>1, j \in \mathbb{N} .
$$

In order to construct the linear operator $T$, we next define isometries $M_{m}$ of the Banach space $L_{q}(0,1)$ by $M_{m} g(t):=g^{*}(m t), m \in \mathbb{N}$, in the notation of (9). Notice that $M_{m} e_{n}=e_{m n}$. 
Lemma 7. The maps $M_{m}$ are isometric linear transformations of $L_{q}(0,1)$ for all $m \in \mathbb{N}$ and $q>1$.

Proof.

$$
\begin{aligned}
& \int_{0}^{1}\left|M_{m} g(t)\right|^{q} \mathrm{~d} t=\int_{0}^{1}\left|g^{*}(m t)\right|^{q} \mathrm{~d} t \\
& =\frac{1}{m} \int_{0}^{m}\left|g^{*}(u)\right|^{q} \mathrm{~d} u=\frac{1}{m} \sum_{k=1}^{m} \int_{k-1}^{k}\left|g^{*}(u)\right|^{q} \mathrm{~d} u \\
& =\frac{1}{m} \sum_{k=1}^{m} \int_{0}^{1}|g(u)|^{q} \mathrm{~d} u=\int_{0}^{1}|g(t)|^{q} \mathrm{~d} t .
\end{aligned}
$$

We now define $T: L_{q}(0,1) \longrightarrow L_{q}(0,1)$ by

$$
T g(t)=\sum_{m=1}^{\infty} \tau_{m} M_{m} g(t)
$$

The above lemma, the triangle inequality and (12) ensure that $T$ is a bounded everywhere-defined operator with $\|T\|_{\left(L_{q} \rightarrow L_{q}\right)} \leqslant \frac{\pi_{p}}{2}$.

We conclude this section by showing that $T e_{n}=f_{n}$. Indeed, by virtue of (10),

$$
T e_{n}=\sum_{m=1}^{\infty} \tau_{m} e_{m n}=\sum_{m-\text { odd }} \widehat{f}_{1}(m) e_{m n}=\sum_{k=1}^{\infty} \widehat{f_{n}}(k) e_{k}=f_{n}
$$

\section{Bounded INVERTIBILITY OF $T$}

In this section we complete the proof of our main result by showing that $T$ has a bounded inverse for all $p \geqslant \frac{12}{11}$ and $1<q<\infty$.

Observe that (13), Lemma 7 and the triangle inequality give

$$
\left\|T-\tau_{1} M_{1}\right\|_{\left(L_{q} \rightarrow L_{q}\right)} \leq \sum_{j=3}^{\infty}\left|\tau_{j}\right| .
$$

Since $M_{1}=I$, [11, p. 196] shows that it suffices to prove

$$
\sum_{j=3}^{\infty}\left|\tau_{j}\right|<\left|\tau_{1}\right| .
$$

We will carry out the verification of (14) separately for $p \geqslant 2$ and $p<2$.

Case $1(p \geqslant 2)$. By virtue of (12) and Lemma 2,

$$
\sum_{j=3}^{\infty}\left|\tau_{j}\right|=\sum_{3 \leqslant j \text { odd }}\left|\tau_{j}\right|<\frac{4 \pi_{2}}{\pi^{2}}\left(\frac{\pi^{2}}{8}-1\right)<\frac{8}{\pi^{2}} .
$$

On the other hand, Lemma 3 ensures that

$$
\begin{aligned}
\tau_{1} & =2 \int_{0}^{1} S_{p}\left(\pi_{p} t\right) \sin \pi t \mathrm{~d} t=4 \int_{0}^{1 / 2} S_{p}\left(\pi_{p} t\right) \sin \pi t \mathrm{~d} t \\
& >4 \int_{0}^{1 / 2} 2 t \sin \pi t \mathrm{~d} t=\frac{8}{\pi^{2}} .
\end{aligned}
$$

This guarantees (14). 


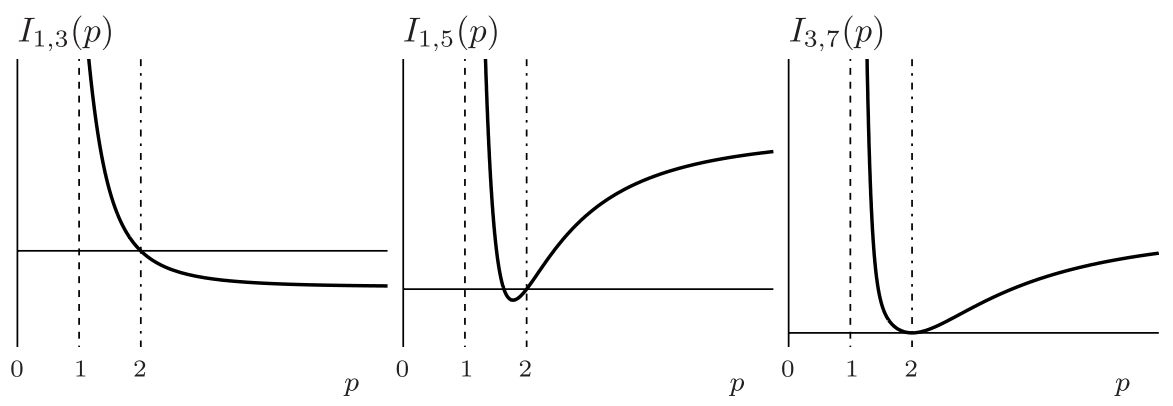

FIGURE 2.

$\underline{\text { Case } 2}\left(\frac{12}{11} \leqslant p<2\right)$. By virtue of Lemma 5 ,

$$
\tau_{1}=2 \int_{0}^{1} S_{p}\left(\pi_{p} t\right) \sin \pi t \mathrm{~d} t>2 \int_{0}^{1}(\sin \pi t)^{2} \mathrm{~d} t=1 .
$$

On the other hand, (11), Lemma 2 and the Cauchy-Schwartz inequality yield

$$
\begin{aligned}
\left|\tau_{j}\right| & =\left|\frac{4 \pi_{p}}{j^{2} \pi^{2}} \int_{0}^{1 / 2} S_{p}^{\prime \prime}\left(\pi_{p} t\right) \sin j \pi t \mathrm{~d} t\right| \\
& \leqslant \frac{4 \pi_{12 / 11}}{j^{2} \pi^{2}}\left(\int_{0}^{1 / 2}\left(S_{p}^{\prime \prime}\left(\pi_{p} t\right)\right)^{2} \mathrm{~d} t\right)^{1 / 2}\left(\int_{0}^{1 / 2}(\sin j \pi t)^{2} \mathrm{~d} t\right)^{1 / 2} .
\end{aligned}
$$

Then (44) and (6) give

$$
\sum_{j=3}^{\infty}\left|\tau_{j}\right| \leqslant \frac{22}{3(\sqrt{3}-1) \pi}\left(\frac{\pi^{2}}{8}-1\right)<1
$$

This completes the proof of Theorem 1 .

Remark 8. A more precise but less explicit statement of Theorem 1 can be given as follows:

There exists $p_{0} \in\left(1, \frac{12}{11}\right)$ such that for $p_{0} \leqslant p<\infty$, the family $\left\{f_{n}\right\}_{n=1}^{\infty}$ forms a Riesz basis of $L_{2}(0,1)$ and a Schauder basis of $L_{q}(0,1)$ for all $1<q<\infty$.

As already mentioned at the end of Section 1 the value $p=\frac{12}{11}$ is by no means the optimal one. For example, our proof already shows that inequality (14) holds also for $p \in\left(\frac{12}{11}-\varepsilon, \frac{12}{11}\right)$ with $\varepsilon>0$ small enough.

Numerical results indicate that (14) holds when $p=1.05$, but not when $p=1.04$, so the method here will not suffice for all $p>1$.

Remark 9. It is obvious that $\left\{f_{n}\right\}_{n=1}^{\infty}$ is not an orthogonal basis in $L^{2}(0,1)$ for $p \neq 2$. Indeed, the sign of

$$
I_{i, j}=\int_{0}^{1} f_{i}(t) f_{j}(t) \mathrm{d} t, \quad i, j=1,2, \ldots
$$

depends on $p$ and can change as $p$ varies from 1 to $\infty$. Figure 2 illustrates the dependence of $I_{1,3}, I_{1,5}$ and $I_{3,7}$ on $p$.

More values of $I_{i, j}$, and further numerical experiments with the bases $\left\{f_{n}\right\}$, can be found at http://drift.cam.zcu.cz. 


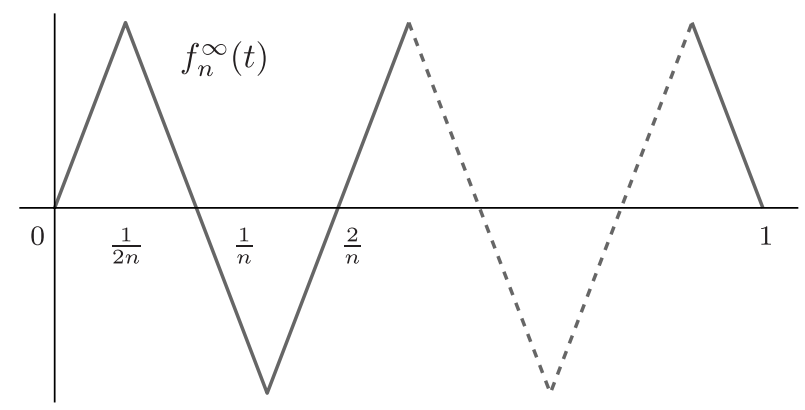

FIGURE 3.

\section{The Limit AS $p \rightarrow \infty$}

Although (11) does not represent a differential equation when $p=\infty$, we can still discuss the pointwise limits $f_{n}^{\infty}$ of the $f_{n}$ as $p \rightarrow \infty$, constructed as follows. On $\left[0, \frac{2}{n}\right)$ we set

$$
f_{n}^{\infty}(t):= \begin{cases}2 n t, & t \in\left[0, \frac{1}{2 n}\right], \\ 2-2 n t, & t \in\left(\frac{1}{2 n}, \frac{3}{2 n}\right), \\ 2 n t-4, & t \in\left[\frac{3}{2 n}, \frac{2}{n}\right),\end{cases}
$$

and extend it periodically to the intervals $\left[\frac{2 k}{n}, \frac{2(k+1)}{n}\right), k \in \mathbb{Z}$. The restriction to $[0,1]$ is a piecewice linear function whose graph is depicted in Figure 3.

Similarly to Section 3 we can define $\tau_{j}^{\infty}:=\widehat{f_{1}^{\infty}}(j)$. As before, $\tau_{j}^{\infty}=0$ when $j$ is even. For odd $j$, integration by parts yields

$$
\begin{aligned}
& \tau_{j}^{\infty}=2 \int_{0}^{1} f_{1}^{\infty}(t) \sin (j \pi t) \mathrm{d} t=4 \int_{0}^{1 / 2} f_{1}^{\infty}(t) \sin (j \pi t) \mathrm{d} t \\
& =\frac{4}{j \pi} \int_{0}^{1 / 2} 2 \cos (j \pi t) \mathrm{d} t=(-1)^{j} \frac{8}{j^{2} \pi^{2}}
\end{aligned}
$$

so

$$
\left|\tau_{j}^{\infty}\right|=\frac{8}{j^{2} \pi^{2}}
$$

Hence, as in Section 4, we have

$$
\sum_{j=3}^{\infty}\left|\tau_{j}^{\infty}\right|<\frac{8}{\pi^{2}}
$$

and so

$$
\sum_{j=3}^{\infty}\left|\tau_{j}^{\infty}\right|<\left|\tau_{1}^{\infty}\right| .
$$

The analogue of Theorem 1 for $p=\infty$ follows as for $p<\infty$, the analogue $T^{\infty}$ : $L_{q}(0,1) \rightarrow L_{q}(0,1)$ of $T$ being defined via

$$
T^{\infty} g(t)=\sum_{m=1}^{\infty} \tau_{m}^{\infty} M_{m} g(t) .
$$


Remark 10. The results of this paper appear to be useful in numerical approaches to boundary value problems for quasilinear equations involving the $p$-Laplacian, e.g., of the type considered in [3] and [4. We hope to report on this elsewhere.

\section{REFERENCES}

[1] C. Bennewitz, Y. SAitō. "An embedding norm and the Lindqvist trigonometric functions", Electronic Journal of Differential Equations 86 (2002), 1-6. MR1938382 (2003j:46028)

[2] P. Binding, P. DrábeK. "Sturm-Liouville theory for the $p$-Laplacian", Studia Scientiarium Mathematicarum Hungarica 40 (2003), 375 - 396. MR2037324 (2004j:34068)

[3] M. Del Pino, P. Drábek and R. F. Manásevich, The Fredholm alternative at the first eigenvalue for the one-dimensional p-Laplacian, J. Differential Equations, 151 (1999), 386419. MR1669705 (99m:34042)

[4] P. Drábek, P. Girg and R. F. Manásevich, Generic Fredholm alternative for the one dimensional p-Laplacian, Nonlin. Diff. Equations and Applications 8 (2001), 285-298. MR.1841260 (2002f:34027)

[5] W. Eberhart, Á. Elbert, "On the eigenvalues of a half-linear boundary value problem", Math. Nachr. 213 (2000), 57 - 76. MR1755246 (2001b:34035)

[6] Á. Elbert. "A half-linear second order differential equation". Coll. Math. Soc. J. Bolyai 30 (1979) 153 - 179. MR0680591 (84g:34008)

[7] Á. Elbert, K. TakÂsı, T. TinagaWa, "An Oscillatory half-linear differential equation", Arch. Math. 33 (1997), 355 - 361. MR1601353(98m:34071)

[8] C. FABRY, D. FAYYAD, "Periodic solutions of second order differential equations with a $p$ Laplacian and asymmetric nonlinearities", Rend. Ist. Mat. Univ. Trieste 24 (1992), $207-$ 227. MR.1310080 (96b:34027)

[9] I. Gohberg, M. KREIN, Introduction to the Theory of Linear Nonselfadjoint Operators. Translations of Mathematical Monographs, Vol. 18 American Mathematical Society, 1969. MR 0246142 (39:7447)

[10] J.R. Higgins, Completeness and the Basis Properties of Sets of Special Functions, Cambridge Tracts in Mathematics, Vol. 72. Cambridge University Press, 1977. MR0499341 (58:17240)

[11] T. Kato, Perturbation Theory for Linear Operators, Springer-Verlag, 1976. MR0407617 $(53: 11389)$

[12] P. Lindqvist, "Note on a nonlinear eigenvalue problem", Rocky Mount. J. Math. 23 (1993), 281 - 288. MR 1212743 (94d:34031)

[13] P. Lindqvist, "Some remarkable sine and cosine functions", Ricerche di Matematica 44 (1995), 269 - 290. MR1469702 (99g:33001)

[14] Y. Naito, "Uniqueness of positive solutions of quasilinear differential equations", Diff. Int. Equations 8 (1995), 1813 - 1822. MR1347982 (96g:34039)

[15] M. Ôtani, "A Remark on certain nonlinear elliptic equations", Proc. Fac. Sci. Tokai Univ. 19 (1984), 23 - 28. MR0753635(86c:35009)

[16] W. Reichel, W. Walter, "Sturm-Liouville type problems for the $p$-Laplacian under asymptotic nonresonance conditions", J. Differential Equations 156 (1999), 50 - 70. MR:1701814 (2000e:34036)

[17] I. Singer, Bases in Banach Spaces I, Springer-Verlag, 1970. MR0298399 (45:7451)

Department of Mathematics and Statistics, University of Calgary, Calgary, AlBerta, Canada T2N 1N4

Department of Mathematics and Statistics, University of Calgary, Calgary, AlBerta, CANada T2N 1N4

Department of Mathematics, University of West Bohemia, Pilsen, Czech Republic

Department of Mathematics, University of West Bohemia, Pilsen, Czech Republic

Department of Mathematics, University of West Bohemia, Pilsen, Czech Republic 